\title{
LADDER THEOREM AND LENGTH-SCALE ESTIMATES FOR A LERAY ALPHA MODEL OF TURBULENCE*
}

\author{
HANI $\mathrm{ALI}^{\dagger}$
}

\begin{abstract}
In this paper, we study the Modified Leray alpha model with periodic boundary conditions. We show that the regular solution satisfies a sequence of energy inequalities which are called "ladder inequalities". Furthermore, we estimate some quantities of physical relevance in terms of the Reynolds number.
\end{abstract}

Key words. Turbulence models, regularity, Navier-Stokes equations.

MSC Classification. 76B03, 76F05, 76D05, 35Q30.

\section{Introduction}

We consider in this paper the ML- $\alpha$ model of turbulence

$$
\left\{\begin{array}{l}
\frac{\partial \boldsymbol{u}}{\partial t}+(\boldsymbol{u} \cdot \nabla) \overline{\boldsymbol{u}}-\nu \Delta \boldsymbol{u}+\nabla p=\boldsymbol{f} \quad \text { in } \mathbb{R}^{+} \times \mathbb{T}_{3}, \\
-\alpha^{2} \Delta \overline{\boldsymbol{u}}+\overline{\boldsymbol{u}}=\boldsymbol{u} \quad \text { in } \mathbb{T}_{3}, \\
\nabla \cdot \boldsymbol{u}=\nabla \cdot \overline{\boldsymbol{u}}=0 \\
\oint_{\mathbb{T}_{3}} \boldsymbol{u}=\oint_{\mathbb{T}_{3}} \overline{\boldsymbol{u}}=0, \\
\boldsymbol{u}_{t=0}=\boldsymbol{u}^{i n}
\end{array}\right.
$$

where the boundary conditions are periodic. Therefore we consider these equations on the three dimensional torus $\mathbb{T}_{3}=\left(\mathbb{R}^{3} / \mathcal{T}_{3}\right)$ where $\mathcal{T}_{3}=2 \pi \mathbb{Z}^{3} / L, \mathbf{x} \in \mathbb{T}_{3}$, and $\left.t \in\right] 0,+\infty[$. The unknowns are the velocity vector field $\boldsymbol{u}$ and the scalar pressure p. The viscosity $\nu$, the initial velocity vector field $\boldsymbol{u}^{i n}$, and the external force $\boldsymbol{f}$ with $\nabla \cdot \boldsymbol{f}=0$ are given. In this paper the force $f$ does not depend on time.

This model was first studied in [8], where the authors prove the global existence and uniqueness of the solution. They also prove the existence of a global attractor $\mathcal{A}$ to this model and they made estimates of the fractal dimension of this attractor in terms of Grashof number $G r$.

The dimension of the attractor gives us some idea of the level of the complexity of the flow. The relation between the number of determining modes, determining nodes, and the evolution of volume elements of the attractors are discussed by Jones and Titi in [12]. Temam also interprets in his book [15] the dimension of the attractors as the number of degrees of freedom of the flow.

It is easily seen that when $\alpha=0$, Equation (1.1) reduce to the usual Navier-Stokes equations for incompressible fluids.

Assuming that $f \in C^{\infty}$, any $C^{\infty}$ solution to the Navier-Stokes equations formally satisfies what is called the ladder inequality [3]. That means that for any $C^{\infty}$ solution $(\boldsymbol{u}, p)$ to the (NSE), the velocity part $\boldsymbol{u}$ satisfies the following relation between its

${ }^{*}$ Received: April 30, 2011; accepted (in revised version): July 19, 2011. Communicated by Alexander Kiselev.

${ }^{\dagger}$ IRMAR, UMR 6625, Université Rennes 1, Campus Beaulieu, 35042 Rennes cedex FRANCE (hani.ali@univ-rennes1.fr). 
higher derivatives:

$$
\begin{aligned}
\frac{1}{2} \frac{d}{d t} H_{N} & \leq-\nu H_{N+1}+C_{N} H_{N}\|\nabla \boldsymbol{u}\|_{\infty}+H_{N}^{1 / 2} \Phi_{N}{ }^{1 / 2}, \\
\text { where } H_{N} & =\int_{\mathbb{T}_{3}}\left|\nabla^{N} \boldsymbol{u}\right|^{2} d \mathbf{x} \text { and } \Phi_{N}=\int_{\mathbb{T}_{3}}\left|\nabla^{N} \boldsymbol{f}\right|^{2} d \mathbf{x} .
\end{aligned}
$$

These differential inequalities are used first in [3] to show the existence of a lower bound on the smallest scale in the flow. The same result is obtained in [4] by a Gevrey class estimate. Recently, the ladder inequalities have been used to study the intermittency of solutions to the Navier-Stokes equations; see [9]. The ladder inequalities to the Navier-Stokes equations are based on the assumption that a solution exists such that the higher order norms are finite. Note that we need no such assumption in the case of the alpha regularization, where existence and uniqueness of a $C^{\infty}$ solution are guaranteed.

The ladder inequalities are generalized in $[10,11]$ to other equations based on the Navier-Stokes equations such as Navier-Stokes-alpha model [5] and Leray alpha model [1].

In this paper we aim to study ladder inequalities for model (1.1). In the whole paper, $\alpha>0$ is given and we assume that the initial data is $C^{\infty}$. One of the main results of this paper is

Theorem 1.1. Assume $\boldsymbol{f} \in C^{\infty}\left(\mathbb{T}_{3}\right)^{3}$ and $\boldsymbol{u}^{\text {in }} \in C^{\infty}\left(\mathbb{T}_{3}\right)^{3}$. Let $(\boldsymbol{u}, p):=\left(\boldsymbol{u}^{\alpha}, p^{\alpha}\right)$ be the unique solution to problem (1.1). Then the velocity part $\boldsymbol{u}$ satisfies the ladder inequalities,

$$
\begin{aligned}
\frac{1}{2}\left(\frac{d}{d t} \overline{H_{N}}+\alpha^{2} \frac{d}{d t} \overline{H_{N+1}}\right) \leq-\nu\left(\overline{H_{N+1}}+\alpha^{2} \overline{H_{N+2}}\right) & \\
& +C_{N}\|\nabla \overline{\boldsymbol{u}}\|_{\infty}\left(\overline{H_{N}}+\alpha^{2} \overline{H_{N+1}}\right)+{\overline{H_{N}}}^{1 / 2} \Phi_{N}{ }^{1 / 2},
\end{aligned}
$$

where

$$
\overline{H_{N}}=\int_{\mathbb{T}_{3}}\left|\nabla^{N} \overline{\boldsymbol{u}}\right|^{2} d \boldsymbol{x}, C_{0}=0 \text { and } C_{N} \approx 2^{N} \text { for all } N \geq 1
$$

The gradient symbol $\nabla^{N}$ here refers to all derivatives of every component of $\boldsymbol{u}$ of order $N$ in $L^{2}\left(\mathbb{T}_{3}\right)$.

REMark 1.1. We note that $\overline{H_{N}} \rightarrow H_{N}$ as $\alpha \rightarrow 0$. Thus we find the inequality (1.2).

Another task of this paper is to estimate quantities of physical relevance in terms of the Reynolds number Re (see (2.8) below); these results are summarized in Table 2.1. For simplicity, Equation (1.1) will be considered with forcing $\boldsymbol{f}(\boldsymbol{x})$ taken to be in $L^{2}$ of narrow band type with a single length scale $\ell$ (see $[9,10]$ ) such that

$$
\left\|\nabla^{n} \boldsymbol{f}\right\|_{L^{2}} \approx \ell^{-n}\|\boldsymbol{f}\|_{L^{2}}
$$

In order to estimate small length scales associated with higher order moments, we combine in Section 5 the force with the higher derivative of the velocity $[3,9,10]$, such that

$$
J_{N}=\overline{F_{N}}+2 \alpha^{2} \overline{F_{N+1}}
$$


where

$$
\overline{F_{N}}=\overline{H_{N}}+\tau^{2} \Phi_{N},
$$

the quantity $\overline{F_{N}}$ is different from 0 , and the quantity $\tau$ is defined by

$$
\tau=\ell^{2} \nu^{-1}(G r \ln G r)^{-1 / 2} \text {. }
$$

We notice that $\tau$ has the dimension of time and is chosen in this way to ensure that the additional forcing term in (1.7) becomes negligible with respect to $\overline{H_{N}}$ when $G r \rightarrow \infty$ (compare [10, Appendix A.2]). The $J_{N}$ is used to define a set of time-dependent inverse length scales

$$
\kappa_{N, r}=\left(\frac{J_{N}}{J_{r}}\right)^{\frac{1}{2(N-r)}} .
$$

The second main result of the paper is the following theorem.

TheOREM 1.2. Let $\boldsymbol{f} \in C^{\infty}\left(\mathbb{T}_{3}\right)^{3}$ be of narrow-band type and $\boldsymbol{u}^{\text {in }} \in C^{\infty}\left(\mathbb{T}_{3}\right)^{3}$. Let $\boldsymbol{u}:=\boldsymbol{u}^{\alpha}$ be the velocity part of the solution to problem (1.1). Then estimates in terms of Reynolds number Re for the length scales associated with higher order moments solution $\kappa_{N, 0}(N \geq 2)$, the inverse Kolmogorov length $\lambda_{k}$, and the attractor dimension $d_{F, M L-\alpha}(\mathcal{A})$ are given by

$$
\begin{gathered}
\ell^{2}\left\langle\kappa_{N, 0}^{2}\right\rangle \leq C(\alpha, \nu, \ell, L)^{(N-1) / N} R e^{5 / 2-3 / 2 N}(\ln R e)^{1 / N}+C R e \ln R e \\
\ell \lambda_{k}^{-1} \leq c R e^{5 / 8} \\
d_{F, M L-\alpha}(\mathcal{A}) \leq c\left(\frac{L^{3} \ell^{-4}}{\alpha^{2} \lambda_{1}^{3 / 2}}\right)^{3 / 4} R e^{9 / 4}
\end{gathered}
$$

where $\langle\cdot\rangle$ is the long time average defined below (2.2).

The paper is organized as follows: In Section 2, we start by summarizing and discussing the results given above. In Section 3 we recall some helpful results about existence and uniqueness for this $\mathrm{ML}-\alpha$ model, and we prove a general regularity result. In Section 4, we prove Theorem 1.1. We stress that for all $N \in \mathbb{N}$ fixed, inequality (1.3) goes to inequality (1.2) when $\alpha \rightarrow 0$, at least formally. In Section 5, we prove Theorem 1.2.

\section{Summary and discussion of the results}

Generally the most important of the estimates in Navier-Stokes theory have been found in terms of the Grashof number $G r$ defined below in terms of the forcing, but these are difficult to compare with the results of Kolmogorov scaling theories [7] which are expressed in terms of Reynolds number $R e_{\mathcal{N S}}$ based on the Navier-Stokes velocity $\boldsymbol{u}$. A good definition of this is

$$
R e_{\mathcal{N S}}=\frac{U \ell}{\nu}, \quad U^{2}=L^{-3}\left\langle\|\boldsymbol{u}\|_{L^{2}}^{2}\right\rangle
$$

where $\langle\cdot\rangle$ is the long time average

$$
\langle g(\cdot)\rangle=\operatorname{Lim}_{t \rightarrow \infty} \frac{1}{t} \int_{0}^{t} g(s) d s,
$$


where Lim indicates a generalized limit that extends the usual limits [6].

With $f_{r m s}=L^{-3 / 2}\|\boldsymbol{f}\|_{L^{2}}$, the standard definition of the Grashof number in three dimensions is

$$
G r=\frac{\ell^{3} f_{r m s}}{\nu^{2}}
$$

Doering and Foias [2] have addressed the problem of how to relate $R e_{\mathcal{N S}}$ and $G r$ and have shown that in the limit $G r \rightarrow \infty$, solutions of the Navier-Stokes equations must satisfy

$$
G r \leq c\left(R e_{\mathcal{N S}}^{2}+R e_{\mathcal{N S}}\right) .
$$

Using the above relation (2.7), Doering and Gibbon [9] have reexpressed some Navier-Stokes estimates in terms of $R e_{\mathcal{N S}}$. In particular they showed that the energy dissipation rate $\epsilon_{\mathcal{N S}}=\nu\left\langle\|\nabla \boldsymbol{u}\|_{L^{2}}^{2}\right\rangle L^{-3}$ is bounded above by

$$
\epsilon_{\mathcal{N S}} \leq c \nu^{3} \ell^{-4}\left(R e_{\mathcal{N S}}^{3}+R e_{\mathcal{N S}}\right)
$$

and the inverse Kolmogorov length $\lambda_{k, \mathcal{N S}}^{-1}=\left(\epsilon_{\mathcal{N S}} / \nu^{3}\right)^{1 / 4}$ is bounded above by

$$
\ell \lambda_{k, \mathcal{N S}}^{-1} \leq c R e_{\mathcal{N} \mathcal{S}}^{3 / 4}
$$

The relation (2.4) is essentially a Navier-Stokes result. In [10] it was shown that this property holds for the Navier-Stokes-alpha model [5]; the same methods can be used to show that for Equation (1.1) we have (compare [10, Appendix A.1])

$$
G r \leq c\left(R e^{2}+R e\right),
$$

where $R e$ is the Reynolds number based on the smoothed velocity $\overline{\boldsymbol{u}}$ which is the solution for Equation (1.1),

$$
R e=\frac{\overline{U \ell}}{\nu}, \bar{U}^{2}=L^{-3}\left\langle\|\overline{\boldsymbol{u}}\|_{L^{2}}^{2}\right\rangle .
$$

In this paper, we will use (2.7) to obtain estimates in terms of the Reynolds number $R e$. These estimates are listed in Table 2.1.

The estimate for $d_{F, M L-\alpha}(\mathcal{A})$ is consistent with the long-standing belief that $R e^{3 / 4} \times R e^{3 / 4} \times R e^{3 / 4}$ resolution grid points are needed to numerically resolve the flow. The fact that this bound is not valid for the Navier-Stokes equations is consistent with the fact that $d_{F, M L-\alpha}(\mathcal{A})$ blows up as $\alpha$ tends to zero. The improved estimate to the inverse Kolmogorov $\lambda_{k}^{-1}$ coincide with the estimate to the Navier-Stokes-alpha given in [10] and blows up when $\alpha$ tends to zero. The estimate for $\left\langle\overline{H_{3}}\right\rangle$ is not calculated for the NS- $\alpha$ and the Bardina model in [10,11], but it can be obtained for these two models by following the same method used here for the Modified Leray$\alpha$. The estimate for $\left\langle\kappa_{N, 0}^{2}\right\rangle$ is obtained directly from the $\|\nabla \overline{\boldsymbol{u}}\|_{\infty}$ term in the ladder inequalities (1.3) as opposed to the $\nu^{-1}\|\overline{\boldsymbol{u}}\|_{\infty}^{2}$ term in [10]. In the case when the ladder inequalities with the $\nu^{-1}\|\overline{\boldsymbol{u}}\|_{\infty}^{2}$ term as in [10] are used, we obtain that the solution to the Modified Leray- $\alpha$ also satisfies $\ell^{2}\left\langle\kappa_{N, 0}^{2}\right\rangle \leq c R e^{\frac{11}{4}-\frac{7}{4 N}}(\ln R e)^{\frac{1}{N}}$. However, the exponent $\frac{5}{2}-\frac{3}{2 N}$ from our study is less than $\frac{11}{4}-\frac{7}{4 N}$ for all $N>1$.

We finish this section with the following remark. The existence and the uniqueness of a $C^{\infty}$ solution for all time $T$ to the ML- $\alpha$ motivate the present study. Provided that a regular solution exists for a maximal interval time $\left[0, T^{*}[\right.$, we can show the ladder inequalities to the Navier-Stokes equations in $\left[0, T^{*}[\right.$. We then naturally ask ourselves if we can use the convergence of (1.3) to (1.2) in $\left[0, T^{*}\right.$ [ to deduce some information about the regular solution beyond the time $T^{*}$. This is a crucial problem. 


\begin{tabular}{|l|c|c|c|c|}
\hline & NS- $\alpha /$ Bardina & Leray- $\alpha$ & ML- $\alpha$ & Eq. \\
\hline \hline$\ell \lambda_{k}^{-1}$ & $R e^{5 / 8}$ & $R e^{7 / 12}$ & $R e^{5 / 8}$ & $(5.23)$ \\
$\left\langle\overline{H_{1}}\right\rangle$ & $R e^{5 / 2}$ & $R e^{7 / 3}$ & $R e^{5 / 2}$ & $(5.22)$ \\
$\left\langle\overline{H_{2}}\right\rangle$ & $R e^{3}$ & $R e^{8 / 3}$ & $R e^{3}$ & $(5.17)$ \\
$\left\langle\overline{H_{3}}\right\rangle$ & $-/-$ & $R e^{3}$ & $R e^{7}$ & $(5.8)$ \\
$d_{F}(\mathcal{A})$ & $R e^{9 / 4} / R e^{9 / 5}$ & $R e^{9 / 7}$ & $R e^{9 / 4}$ & $(5.25)$ \\
$\ell^{2}\left\langle\kappa_{N, r}^{2}\right\rangle$ & $R e^{11 / 4}$ & $R e^{17 / 12}$ & $R e^{5 / 2}$ & $(5.10)$ \\
$\ell^{2}\left\langle\kappa_{1,0}^{2}\right\rangle$ & $R e \ln R e$ & $R e \ln R e$ & $R e \ln R e$ & $(5.5)$ \\
$\left\langle\|\overline{\boldsymbol{u}}\|_{\infty}^{2}\right\rangle$ & $R e^{11 / 4}$ & $R e^{5 / 2}$ & $R e^{11 / 4}$ & $(5.6)$ \\
$\left\langle\|\nabla \overline{\boldsymbol{u}}\|_{\infty}\right\rangle$ & $R e^{35 / 16}$ & $R e^{17 / 12}$ & $R e^{5 / 2}$ & $(5.9)$ \\
$\ell^{2}\left\langle\kappa_{N, 0}^{2}\right\rangle$ & $R e^{\frac{11}{4}-\frac{7}{4 N}}(\ln R e)^{\frac{1}{N}}$ & $R e^{\frac{17}{12}-\frac{5}{12 N}}(\ln R e)^{\frac{1}{N}}$ & $R e^{\frac{5}{2}-\frac{3}{2 N}}(\ln R e)^{\frac{1}{N}}$ & $(5.13)$ \\
\hline
\end{tabular}

TABLE 2.1. Comparison of various upper bounds for the Navier-Stokes- $\alpha$, Bardina, Leray$\alpha$ from [10, 11], and Modified Leray- $\alpha$ with constant omitted.

\section{Existence, unicity and regularity results}

We begin this section by recalling the system (1.1) considered with periodic boundary conditions.

$$
\left\{\begin{array}{l}
\frac{\partial \boldsymbol{u}}{\partial t}+(\boldsymbol{u} \cdot \nabla) \overline{\boldsymbol{u}}-\nu \Delta \boldsymbol{u}+\nabla p=\boldsymbol{f} \quad \text { in } \mathbb{R}^{+} \times \mathbb{T}_{3}, \\
-\alpha^{2} \Delta \overline{\boldsymbol{u}}+\overline{\boldsymbol{u}}=\boldsymbol{u} \quad \text { in } \mathbb{T}_{3}, \\
\nabla \cdot \boldsymbol{u}=\nabla \cdot \overline{\boldsymbol{u}}=0 \\
\oint_{\mathbb{T}_{3}} \boldsymbol{u}=\oint_{\mathbb{T}_{3}} \overline{\boldsymbol{u}}=0 \\
\boldsymbol{u}_{t=0}=\boldsymbol{u}^{i n}
\end{array}\right.
$$

Note that given $\boldsymbol{u}=\overline{\boldsymbol{u}}-\alpha^{2} \Delta \overline{\boldsymbol{u}}$, the Poincaré inequality $\|\boldsymbol{u}\|_{L^{2}} \leq L / 2 \pi\|\nabla \boldsymbol{u}\|_{L^{2}}$ immediately leads to

$$
\alpha^{2}\|\overline{\boldsymbol{u}}\|_{H^{2}} \leq\|\boldsymbol{u}\|_{L^{2}} \leq\left(\frac{L^{2}}{4 \pi^{2}}+\alpha^{2}\right)\|\overline{\boldsymbol{u}}\|_{H^{2}} .
$$

In order to prove the ladder inequalities (1.3) we need first to show a regularity result for (1.1) or (3.1).

Proposition 3.1. If $\boldsymbol{f} \in H^{m-1}\left(\mathbb{T}_{3}\right)^{3}$ and $\boldsymbol{u}^{\text {in }} \in H^{m}\left(\mathbb{T}_{3}\right)^{3}, m \geq 1$, then the solution $(\boldsymbol{u}, p)$ of (1.1) is such that

$$
\begin{aligned}
& \boldsymbol{u} \in L^{\infty}\left([0, T], H^{m}\left(\mathbb{T}_{3}\right)^{3}\right) \cap L^{2}\left([0, T], H^{m+1}\left(\mathbb{T}_{3}\right)^{3}\right), \\
& p \in L^{2}\left([0, T], H^{m}\left(\mathbb{T}_{3}\right)^{3}\right) .
\end{aligned}
$$

The following theorem is a direct consequence of Proposition 3.1.

Theorem 3.1. Assume $\boldsymbol{f} \in C^{\infty}\left(\mathbb{T}_{3}\right)^{3}$ and $\boldsymbol{u}^{i n} \in C^{\infty}\left(\mathbb{T}_{3}\right)^{3}$. Let $(\boldsymbol{u}, p)$ be the solution to problem (1.1). Then the solution is $C^{\infty}$ in space and time.

The aim of this section is the proof of Proposition 3.1. We begin by recalling some known results for (1.1) or (3.1). 
3.1. Known results. Results in [8] can be summarized as follows:

Theorem 3.2. Assume $\boldsymbol{f} \in L^{2}\left(\mathbb{T}_{3}\right)^{3}$ and $\boldsymbol{u}^{\text {in }} \in H^{1}\left(\mathbb{T}_{3}\right)^{3}$. Then for any $T>0$, (1.1) has a unique distributional solution $(\boldsymbol{u}, p):=\left(\boldsymbol{u}^{\alpha}, p^{\alpha}\right)$ such that

$$
\begin{gathered}
\boldsymbol{u} \in L^{\infty}\left([0, T], H^{-1}\left(\mathbb{T}_{3}\right)^{3}\right) \cap L^{2}\left([0, T], L^{2}\left(\mathbb{T}_{3}\right)^{3}\right), \\
\overline{\boldsymbol{u}} \in L^{\infty}\left([0, T], H^{1}\left(\mathbb{T}_{3}\right)^{3}\right) \cap L^{2}\left([0, T], H^{2}\left(\mathbb{T}_{3}\right)^{3}\right), \\
\|\overline{\boldsymbol{u}}(t)\|_{L^{2}}^{2}+\alpha^{2}\|\overline{\boldsymbol{u}}(t)\|_{H^{1}}^{2} \leq\left(\left\|\boldsymbol{u}^{i n}\right\|_{L^{2}}^{2}+\alpha^{2}\left\|\boldsymbol{u}^{i n}\right\|_{H^{1}}^{2}\right) \exp \left(-4 \pi \nu t / L^{2}\right) \\
+\frac{L^{2}}{4 \pi^{2} \nu^{2}}\|\boldsymbol{f}\|_{H^{-1}}^{2}\left(1-\exp \left(-4 \pi \nu t / L^{2}\right)\right) .
\end{gathered}
$$

Furthermore, if $\boldsymbol{u}^{\text {in }} \in H^{2}\left(\mathbb{T}_{3}\right)^{3}$ then

$$
\begin{aligned}
& \boldsymbol{u} \in L^{\infty}\left([0, T], L^{2}\left(\mathbb{T}_{3}\right)^{3}\right), \\
& \overline{\boldsymbol{u}} \in L^{\infty}\left([0, T], H^{2}\left(\mathbb{T}_{3}\right)^{3}\right), \\
& \|\overline{\boldsymbol{u}}(t)\|_{H^{1}}^{2}+\alpha^{2}\|\overline{\boldsymbol{u}}(t)\|_{H^{2}}^{2} \leq k(t), .
\end{aligned}
$$

Where $k(t)$ satisfies

(i) $k(t)$ is finite for all $t>0$.

(ii) $\limsup _{t \rightarrow \infty} k(t)<\infty$.

REMARK 3.1.

(1) The proof is based on the following energy inequality that is obtained by taking the inner product of (1.1) with $\overline{\boldsymbol{u}}$ :

$$
\frac{1}{2}\left(\frac{d}{d t}\|\overline{\boldsymbol{u}}\|_{L^{2}}^{2}+\alpha^{2} \frac{d}{d t}\|\nabla \overline{\boldsymbol{u}}\|_{L^{2}}^{2}\right)+\nu\left(\|\nabla \overline{\boldsymbol{u}}\|_{L^{2}}^{2}+\alpha^{2}\|\Delta \overline{\boldsymbol{u}}\|_{L^{2}}^{2}\right) \leq\|\boldsymbol{f}\|_{L^{2}}\|\overline{\boldsymbol{u}}\|_{L^{2}}
$$

(2) Note that the pressure may be reconstructed from $\boldsymbol{u}$ and $\overline{\boldsymbol{u}}$ by solving the elliptic equation

$$
\Delta p=\nabla \cdot((\boldsymbol{u} \cdot \nabla) \overline{\boldsymbol{u}}) .
$$

One concludes from the classical elliptic theory that $p \in L^{1}\left([0, T], L^{2}\left(\mathbb{T}_{3}\right)^{3}\right)$.

We recall that we can extract subsequences of solutions that converge as $\alpha \rightarrow 0$ to a weak solution of the Navier-Stokes equations. The reader can look in [8] and [5] for more details.

Corollary 3.1.

(1) We have $\boldsymbol{u} \in L^{2}\left([0, T], L^{2}\left(\mathbb{T}_{3}\right)\right)$, and by the Sobolev embedding we obtain that $\overline{\boldsymbol{u}} \in$ $L^{2}\left([0, T], L^{\infty}\left(\mathbb{T}_{3}\right)\right)$. Thus there exists a constant $M(T):=M\left(\boldsymbol{u}^{i n}, \boldsymbol{f}, \alpha, T\right)>0$ such that

$$
\int_{0}^{t}\|\overline{\boldsymbol{u}}\|_{L^{\infty}}^{2} \leq \frac{1}{\alpha^{2}} \int_{0}^{t}\|\boldsymbol{u}\|_{L^{2}}^{2} \leq M(T) \quad \text { for all } t \in[0, T] .
$$

(2) We also observe by using (3.2) that there exists a constant $C(\alpha):=C(\alpha, L)>0$ such that

$$
\|\boldsymbol{u}(t)\|_{L^{2}}^{2} \leq C(\alpha) k(t),
$$

for all $t>0$, and $k(t)$ is defined in (3.10). 
3.2. Regularity: Proof of proposition 3.1. The proof of proposition 3.1 is classical (see for example in [13]). In order to make the paper self-contained we will give a complete proof for this regularity result. The proof is given in many steps.

Step 1: We show that $\boldsymbol{u} \in L^{\infty}\left([0, T], L^{2}\left(\mathbb{T}_{3}\right)^{3}\right) \cap L^{2}\left([0, T], H^{1}\left(\mathbb{T}_{3}\right)^{3}\right)$.

Step 2: We take $\partial_{t} \boldsymbol{u}$ as a test function in (1.1).

Step 3: We take the $m-1$ derivative of (1.1) then we take $\partial_{t} \nabla^{m-1} \boldsymbol{u}$ as a test function and the result follows by induction.

Step 1: We have the following Lemma.

Lemma 3.1. For $\boldsymbol{u}^{\text {in }} \in L^{2}\left(\mathbb{T}_{3}\right)^{3}$ and $\boldsymbol{f} \in H^{-1}\left(\mathbb{T}_{3}\right)^{3}$, Equation (1.1) has a unique solution $(\boldsymbol{u}, p)$ such that

$$
\boldsymbol{u} \in L^{\infty}\left([0, T], L^{2}\left(\mathbb{T}_{3}\right)^{3}\right) \cap L^{2}\left([0, T], H^{1}\left(\mathbb{T}_{3}\right)^{3}\right) .
$$

Proof of Lemma 3.1. We show formal a priori estimates for the solution established in Theorem 3.2. These estimates can be obtained rigorously using the Galerkin procedure.

We take the inner product of (1.1) with $\boldsymbol{u}$ to obtain

$$
\frac{1}{2} \frac{d}{d t}\|\boldsymbol{u}(t, \mathbf{x})\|_{L^{2}}^{2}+\nu\|\nabla \boldsymbol{u}(t, \mathbf{x})\|_{L^{2}}^{2} \leq\left\|\nabla^{-1} \boldsymbol{f}\right\|_{L^{2}}\|\nabla \boldsymbol{u}\|_{L^{2}}+|((\boldsymbol{u} \cdot \nabla) \overline{\boldsymbol{u}}, \boldsymbol{u})| .
$$

Integration by parts and the Cauchy-Schwarz inequality yield

$$
|((\boldsymbol{u} \cdot \nabla) \overline{\boldsymbol{u}}, \boldsymbol{u})| \leq\|\boldsymbol{u} \otimes \overline{\boldsymbol{u}}\|_{L^{2}}\|\nabla \boldsymbol{u}\|_{L^{2}},
$$

and by Young's inequality we obtain

$$
\begin{gathered}
\left\|\nabla^{-1} \boldsymbol{f}\right\|_{L^{2}}\|\nabla \boldsymbol{u}\|_{L^{2}} \leq \frac{1}{\nu}\left\|\nabla^{-1} \boldsymbol{f}\right\|_{L^{2}}^{2}+\frac{\nu}{4}\|\nabla \boldsymbol{u}\|_{L^{2}}^{2}, \\
|((\boldsymbol{u} \cdot \nabla) \overline{\boldsymbol{u}}, \boldsymbol{u})| \leq \frac{1}{\nu}\|\boldsymbol{u} \otimes \overline{\boldsymbol{u}}\|_{L^{2}}^{2}+\frac{\nu}{4}\|\nabla \boldsymbol{u}\|_{L^{2}}^{2} .
\end{gathered}
$$

From the above inequalities we get

$$
\begin{aligned}
\frac{d}{d t}\|\boldsymbol{u}(t, \mathbf{x})\|_{L^{2}}^{2}+\nu\|\nabla \boldsymbol{u}(t, \mathbf{x})\|_{L^{2}}^{2} & \leq \frac{2}{\nu}\left\|\nabla^{-1} \boldsymbol{f}\right\|_{L^{2}}^{2}+\frac{2}{\nu}\|\boldsymbol{u} \overline{\boldsymbol{u}}\|_{L^{2}}^{2} \\
& \leq \frac{2}{\nu}\left\|\nabla^{-1} \boldsymbol{f}\right\|_{L^{2}}^{2}+\frac{2}{\nu} \frac{1}{\alpha^{2}}\|\boldsymbol{u}\|_{L^{2}}^{4},
\end{aligned}
$$

where we have used in the last step that

$$
\|\overline{\boldsymbol{u}}\|_{L^{\infty}}^{2} \leq \frac{1}{\alpha^{2}}\|\boldsymbol{u}\|_{L^{2}}^{2}
$$

This implies that

$$
\frac{d}{d t}\left(1+\|\boldsymbol{u}(t, \mathbf{x})\|_{L^{2}}^{2}\right) \leq C_{1}\left(1+\|\boldsymbol{u}(t, \mathbf{x})\|_{L^{2}}^{2}\right)^{2},
$$

where $C_{1}=\max \left(\frac{2}{\nu} \frac{1}{\alpha^{2}}, \frac{2}{\nu}\left\|\nabla^{-1} \boldsymbol{f}\right\|_{L^{2}}^{2}\right)$. By Grönwall's Lemma, since $\|\boldsymbol{u}\|_{L^{2}}^{2} \in L^{1}([0, T])$ (Corollary 3.1), we conclude that

$$
1+\|\boldsymbol{u}(t, \mathbf{x})\|_{L^{\infty}\left([0, T], L^{2}\right)}^{2} \leq K_{1}(T)
$$


where $K_{1}(T):=K_{1}\left(T, \boldsymbol{u}^{i n}, \boldsymbol{f}\right)$ is given by

$$
K_{1}(T)=\left(1+\left\|\boldsymbol{u}^{i n}\right\|_{L^{2}}^{2}\right) \exp \left(C_{1} \int_{0}^{T}\left(1+\|\boldsymbol{u}(s)\|_{L^{2}}^{2}\right) d s\right) .
$$

Furthermore, for every $T>0$ we have, from (3.17),

$$
\|\boldsymbol{u}(T, \mathbf{x})\|_{L^{2}}^{2}+\nu \int_{0}^{T}\|\nabla \boldsymbol{u}(t, \mathbf{x})\|_{L^{2}}^{2} d t \leq\left\|\boldsymbol{u}^{i n}\right\|_{L^{2}}^{2}+\frac{2}{\nu}\left\|\nabla^{-1} \boldsymbol{f}\right\|_{L^{2}}^{2} T+\frac{2}{\nu} K_{1} M
$$

Thus $\boldsymbol{u} \in L^{2}\left([0, T], H^{1}\left(\mathbb{T}_{3}\right)^{3}\right)$ for all $T>0$.

\section{Step 2:}

With the same assumption in the initial data as in Theorem 3.2 , we can find the following result:

Lemma 3.2. Assume $\boldsymbol{f} \in L^{2}\left(\mathbb{T}_{3}\right)^{3}$ and $\boldsymbol{u}^{\text {in }} \in H^{1}\left(\mathbb{T}_{3}\right)^{3}$. Then for any $T>0$, Equation (1.1) has a unique regular solution $(\boldsymbol{u}, p)$ such that

$$
\begin{aligned}
& \boldsymbol{u} \in C\left([0, T], H^{1}\left(\mathbb{T}_{3}\right)^{3}\right) \cap L^{2}\left([0, T], H^{2}\left(\mathbb{T}_{3}\right)^{3}\right), \\
& \frac{d \boldsymbol{u}}{d t} \in L^{2}\left([0, T], L^{2}\left(\mathbb{T}_{3}\right)^{3}\right), \\
& p \in L^{2}\left([0, T], H^{1}\left(\mathbb{T}_{3}\right)^{3}\right) .
\end{aligned}
$$

Proof of Lemma 3.2. It is easily checked that since $\boldsymbol{u} \in L^{\infty}\left([0, T], L^{2}\left(\mathbb{T}_{3}\right)^{3}\right) \cap$ $L^{2}\left([0, T], H^{1}\left(\mathbb{T}_{3}\right)^{3}\right)$, then $\overline{\boldsymbol{u}} \in L^{\infty}\left([0, T], H^{2}\left(\mathbb{T}_{3}\right)^{3}\right) \cap L^{2}\left([0, T], H^{3}\left(\mathbb{T}_{3}\right)^{3}\right)$. Consequently, by the Sobolev Injection Theorem, we deduce that $\overline{\boldsymbol{u}} \in L^{\infty}\left([0, T], L^{\infty}\left(\mathbb{T}_{3}\right)^{3}\right)$ and $\nabla \overline{\boldsymbol{u}} \in$ $L^{2}\left([0, T], L^{\infty}\left(\mathbb{T}_{3}\right)^{3}\right)$.

Therefore,

$$
(\boldsymbol{u} \cdot \nabla) \overline{\boldsymbol{u}} \in L^{2}\left([0, T], L^{2}\left(\mathbb{T}_{3}\right)^{3}\right) .
$$

Now, for fixed $\mathrm{t}$, we can take $\partial_{t} \boldsymbol{u}$ as a test function in (1.1), and the procedure is the same as the one in [14]. Note that the proof given in [14] is formal and can be obtained rigorously by using the Galerkin method combined with (3.24).

Once we obtain that

$$
\boldsymbol{u} \in L^{\infty}\left([0, T], H^{1}\left(\mathbb{T}_{3}\right)^{3}\right) \cap L^{2}\left([0, T], H^{2}\left(\mathbb{T}_{3}\right)^{3}\right) \cap H^{1}\left([0, T], L^{2}\left(\mathbb{T}_{3}\right)^{3}\right)
$$

and $p \in L^{2}\left([0, T], H^{1}\left(\mathbb{T}_{3}\right)^{3}\right)$, interpolating between $L^{2}\left([0, T], H^{2}\left(\mathbb{T}_{3}\right)^{3}\right)$ and $H^{1}\left([0, T], L^{2}\left(\mathbb{T}_{3}\right)^{3}\right)$ yields $\boldsymbol{u} \in C\left([0, T], H^{1}\left(\mathbb{T}_{3}\right)^{3}\right)$.

\section{Step 3:}

We proceed by induction. The case $m=1$ follows from Lemma 3.2.

Assume that for any $k=1, \ldots, m-1$, if $\boldsymbol{f} \in H^{k-1}\left(\mathbb{T}_{3}\right)^{3}$ and $\boldsymbol{u}^{i n} \in H^{k}\left(\mathbb{T}_{3}\right)^{3}$ then $\boldsymbol{u} \in L^{\infty}\left([0, T], H^{k}\left(\mathbb{T}_{3}\right)^{3}\right) \cap L^{2}\left([0, T], H^{k+1}\left(\mathbb{T}_{3}\right)^{3}\right)$ holds.

It remains to prove that when $k=m, \boldsymbol{f} \in H^{m-1}\left(\mathbb{T}_{3}\right)^{3}$ and $\boldsymbol{u}^{i n} \in H^{m}\left(\mathbb{T}_{3}\right)^{3}$ that $\boldsymbol{u} \in L^{\infty}\left([0, T], H^{m}\left(\mathbb{T}_{3}\right)^{3}\right) \cap L^{2}\left([0, T], H^{m+1}\left(\mathbb{T}_{3}\right)^{3}\right)$.

It is easily checked that for $\boldsymbol{u} \in L^{\infty}\left([0, T], H^{k}\left(\mathbb{T}_{3}\right)^{3}\right) \cap L^{2}\left([0, T], H^{k+1}\left(\mathbb{T}_{3}\right)^{3}\right)$, $\overline{\boldsymbol{u}} \in L^{\infty}\left([0, T], H^{k+2}\left(\mathbb{T}_{3}\right)^{3}\right) \cap L^{2}\left([0, T], H^{k+3}\left(\mathbb{T}_{3}\right)^{3}\right)$. 
Consequently, by the Sobolev Injection Theorem, we deduce that $\nabla^{k} \overline{\boldsymbol{u}} \in L^{\infty}\left([0, T], L^{\infty}\left(\mathbb{T}_{3}\right)^{3}\right)$, and $\nabla^{k+1} \overline{\boldsymbol{u}} \in L^{2}\left([0, T], L^{\infty}\left(\mathbb{T}_{3}\right)^{3}\right)$.

By taking the $m-1$ derivative of (1.1) we get, in the sense of distributions, that

$$
\left\{\begin{array}{l}
\frac{\partial \nabla^{m-1} \boldsymbol{u}}{\partial t}+\nabla^{m-1}((\boldsymbol{u} \cdot \nabla) \overline{\boldsymbol{u}})-\nu \nabla^{m-1} \Delta \boldsymbol{u}+\nabla^{m-1} \nabla p=\nabla^{m-1} \boldsymbol{f} \\
\nabla \cdot \nabla^{m-1} \boldsymbol{u}=0 \\
\nabla^{m-1} \boldsymbol{u}_{t=0}=\nabla^{m-1} \boldsymbol{u}^{i n} .
\end{array}\right.
$$

where boundary conditions remain periodic with zero mean and the initial condition still has zero divergence and mean.

Therefore, after using Leibniz Formula

$$
\nabla^{m-1}((\boldsymbol{u} \cdot \nabla) \overline{\boldsymbol{u}})=\sum_{k=0}^{m-1} C_{m-1}^{k} \nabla^{k} \boldsymbol{u} \nabla^{m-k} \overline{\boldsymbol{u}}
$$

since

$$
\nabla^{k} \boldsymbol{u} \in L^{\infty}\left([0, T], L^{2}\left(\mathbb{T}_{3}\right)^{3}\right)
$$

and

$$
\nabla^{k+1} \overline{\boldsymbol{u}} \in L^{2}\left([0, T], L^{\infty}\left(\mathbb{T}_{3}\right)^{3}\right),
$$

for any $k=1, \ldots, m-1$, we deduce that

$$
\nabla^{m-1}((\boldsymbol{u} \cdot \nabla) \overline{\boldsymbol{u}}) \in L^{2}\left([0, T], L^{2}\left(\mathbb{T}_{3}\right)^{3}\right) .
$$

Now, for fixed t, we can take $\partial_{t} \nabla^{m-1} \boldsymbol{u}$ as a test function in (3.25) and the procedure is the same as the one in [14]. One obtains that $\boldsymbol{u} \in L^{\infty}\left([0, T], H^{m}\left(\mathbb{T}_{3}\right)^{3}\right) \cap$ $L^{2}\left([0, T], H^{m+1}\left(\mathbb{T}_{3}\right)^{3}\right)$ and $p \in L^{2}\left([0, T], H^{m}\left(\mathbb{T}_{3}\right)^{3}\right)$. This finishes the proof of Proposition 3.1 .

\section{Ladder inequalities: Proof of Theorem 1.1}

The first step in the proof of Theorem 1.1, which has been expressed in Section 1 , is the energy inequality (3.11) that corresponds to the case when $N=0$ in the inequality (1.3). Having showed in the above section the regularity result for (1.1), we can take the $N$ derivative of (1.1) and get, in the sense of distributions, that for all $N \geq 1$

$$
\left\{\begin{array}{l}
\frac{\partial \nabla^{N} \boldsymbol{u}}{\partial t}+\nabla^{N}((\boldsymbol{u} \cdot \nabla) \overline{\boldsymbol{u}})-\nu \nabla^{N} \Delta \boldsymbol{u}+\nabla^{N} \nabla p=\nabla^{N} \boldsymbol{f} \\
\nabla \cdot \nabla^{N} \boldsymbol{u}=0 \\
\nabla^{N} \boldsymbol{u}_{t=0}=\nabla^{N} \boldsymbol{u}^{i n}
\end{array}\right.
$$

where the boundary conditions remain periodic with zero mean and the initial condition still has zero divergence and mean. Taking $\nabla^{N} \boldsymbol{u}$ as test function in (4.1), we can write

$$
\begin{aligned}
& \frac{1}{2} \frac{d}{d t} \int_{\mathbb{T}_{3}}\left|\nabla^{N} \overline{\boldsymbol{u}}\right|^{2} d \mathbf{x}+\alpha^{2} \frac{1}{2} \frac{d}{d t} \int_{\mathbb{T}_{3}}\left|\nabla^{N+1} \overline{\boldsymbol{u}}\right|^{2} d \mathbf{x} \\
= & \nu \int_{\mathbb{T}_{3}} \nabla^{N} \overline{\boldsymbol{u}} \nabla^{N} \Delta \overline{\boldsymbol{u}} d \mathbf{x}-\nu \alpha^{2} \int_{\mathbb{T}_{3}} \nabla^{N} \overline{\boldsymbol{u}} \nabla^{N} \Delta \Delta \overline{\boldsymbol{u}} d \mathbf{x} \\
& \quad+\int_{\mathbb{T}_{3}} \nabla^{N} \overline{\boldsymbol{u}} \nabla^{N}((\overline{\boldsymbol{u}} \cdot \nabla) \overline{\boldsymbol{u}}) d \mathbf{x}-\alpha^{2} \int_{\mathbb{T}_{3}} \nabla^{N} \overline{\boldsymbol{u}} \nabla^{N}((\Delta \overline{\boldsymbol{u}} \cdot \nabla) \overline{\boldsymbol{u}}) d \mathbf{x}+\int_{\mathbb{T}_{3}} \nabla^{N} \overline{\boldsymbol{u}} \nabla^{N} \boldsymbol{f} d \mathbf{x},
\end{aligned}
$$


where the pressure term vanishes as $\nabla \cdot \nabla^{N} \boldsymbol{u}=0$.

Using the definition of $\overline{H_{N}}$ in (1.4) we obtain

$$
\begin{aligned}
& \frac{1}{2}\left(\frac{d}{d t} \overline{H_{N}}+\alpha^{2} \frac{d}{d t} \overline{H_{N+1}}\right) \\
\leq & -\nu\left(\overline{H_{N+1}}+\alpha^{2} \overline{H_{N+2}}\right)+\left|\int_{\mathbb{T}_{3}} \nabla^{N} \overline{\boldsymbol{u}} \nabla^{N}((\overline{\boldsymbol{u}} \cdot \nabla) \overline{\boldsymbol{u}}) d \mathbf{x}\right| \\
& +\alpha^{2}\left|\int_{\mathbb{T}_{3}} \nabla^{N+1} \overline{\boldsymbol{u}} \nabla^{N-1}((\Delta \overline{\boldsymbol{u}} \cdot \nabla) \overline{\boldsymbol{u}}) d \mathbf{x}\right|+\left|\int_{\mathbb{T}_{3}} \nabla^{N} \overline{\boldsymbol{u}} \nabla^{N} \boldsymbol{f} d \mathbf{x}\right|,
\end{aligned}
$$

where we have integrated by parts in the Laplacian terms.

The central terms are

$$
\mathrm{NL}_{1}=\left|\int_{\mathbb{T}_{3}} \nabla^{N} \overline{\boldsymbol{u}} \nabla^{N}((\overline{\boldsymbol{u}} \cdot \nabla) \overline{\boldsymbol{u}}) d \mathbf{x}\right|
$$

and

$$
\mathrm{NL}_{2}=\alpha^{2}\left|\int_{\mathbb{T}_{3}} \nabla^{N+1} \overline{\boldsymbol{u}} \nabla^{N-1}((\Delta \overline{\boldsymbol{u}} \cdot \nabla) \overline{\boldsymbol{u}}) d \mathbf{x}\right|
$$

These two terms $\mathrm{NL}_{1}$ and $\mathrm{NL}_{2}$ can be bounded using the following GagliardoNirenberg interpolation inequality [3]:

Lemma 4.1. The Gagliardo-Nirenberg interpolation inequality is:

For $1 \leq q, r \leq \infty, j$ and $m$ such that $0 \leq j<m$ we have

$$
\left\|\nabla^{j} \boldsymbol{v}\right\|_{p} \leq C\left\|\nabla^{m} \boldsymbol{v}\right\|_{r}^{a}\|\boldsymbol{v}\|_{q}^{1-a}
$$

where

$$
\frac{1}{p}=\frac{j}{d}+a\left(\frac{1}{r}-\frac{m}{d}\right)+\frac{1-a}{q}
$$

for $\frac{j}{m} \leq a<1$ and $a=\frac{j}{m}$ if $m-j-\frac{d}{r} \in N^{*}$.

The first nonlinear term $\mathrm{NL}_{1}$ is estimated with the Gagliardo-Nirenberg inequality [3] by $c_{N}\|\nabla \overline{\boldsymbol{u}}\|_{\infty} \overline{H_{N}}$, where $c_{0}=0$ and $c_{N} \leq c 2^{N}$. Indeed, the nonlinear first term $\mathrm{NL}_{1}$ is found to satisfy

$$
\mathrm{NL}_{1}=\left|\int_{\mathbb{T}_{3}} \nabla^{N} \overline{\boldsymbol{u}} \nabla^{N}((\overline{\boldsymbol{u}} \cdot \nabla) \overline{\boldsymbol{u}}) d \mathbf{x}\right| \leq 2^{N}{\overline{H_{N}}}^{1 / 2} \sum_{l=1}^{N}\left\|\nabla^{l} \overline{\boldsymbol{u}}\right\|_{L^{p}}\left\|\nabla^{N+1-l} \overline{\boldsymbol{u}}\right\|_{L^{q}},
$$

where $p$ and $q$ satisfy $1 / p+1 / q=1 / 2$ according to the Hölder inequality.

We use now the two Gagliardo-Nirenberg inequalities

$$
\begin{array}{r}
\left\|\nabla^{l} \overline{\boldsymbol{u}}\right\|_{L^{p}} \leq c_{1}\left\|\nabla^{N} \overline{\boldsymbol{u}}\right\|_{L^{2}}^{a}\|\nabla \overline{\boldsymbol{u}}\|_{\infty}^{1-a}, \\
\left\|\nabla^{N+1-l} \overline{\boldsymbol{u}}\right\|_{L^{q}} \leq c_{2}\left\|\nabla^{N} \overline{\boldsymbol{u}}\right\|_{L^{2}}^{b}\|\nabla \overline{\boldsymbol{u}}\|_{\infty}^{1-b},
\end{array}
$$


where $a$ and $b$ must satisfy

$$
\begin{aligned}
& \frac{1}{p}=\frac{l-1}{3}+a\left(\frac{1}{2}-\frac{N-1}{3}\right), \\
& \frac{1}{q}=\frac{N-l}{3}+b\left(\frac{1}{2}-\frac{N-1}{3}\right) .
\end{aligned}
$$

Since $1 / p+1 / q=1 / 2$, we deduce $a+b=1$. Thus we obtain

$$
\left|\int_{\mathbb{T}_{3}} \nabla^{N} \overline{\boldsymbol{u}} \nabla^{N}((\overline{\boldsymbol{u}} \cdot \nabla) \overline{\boldsymbol{u}}) d \mathbf{x}\right| \leq c_{N}\|\nabla \overline{\boldsymbol{u}}\|_{\infty} \overline{H_{N}} .
$$

In the same way, we can estimate the nonlinear second term with GagliardoNirenberg inequality in order to have

$$
\alpha^{2}\left|\int_{\mathbb{T}_{3}} \nabla^{N+1} \overline{\boldsymbol{u}} \nabla^{N-1}((\Delta \overline{\boldsymbol{u}} \cdot \nabla) \overline{\boldsymbol{u}}) d \mathbf{x}\right| \leq c_{N}^{\prime} \alpha^{2}\|\nabla \overline{\boldsymbol{u}}\|_{\infty} \overline{H_{N+1}},
$$

where $c_{N}^{\prime} \leq c 2^{N}$.

The result (1.3) then follows.

\section{Estimates in terms of Reynolds number: Proof of Theorem 1.2}

5.1. Proof of inequality (1.10). We begin by forming the combination

$$
\overline{F_{N}}=\overline{H_{N}}+\tau^{2} \Phi_{N},
$$

where the quantity $\tau$ is defined by

$$
\tau=\ell^{2} \nu^{-1}(G r \ln G r)^{-1 / 2} .
$$

We define the combination

$$
J_{N}=\overline{F_{N}}+2 \alpha^{2} \overline{F_{N+1}} .
$$

The following result is a consequence of Theorem 1.1 and its proof follows closely to that of the Navier-Stokes-alpha model in [10] and we will not repeat it.

Theorem 5.1. As $G r \rightarrow \infty$, for $N \geq 1,1 \leq p \leq N$ the unique solution to Equation (1.1) satisfies

$$
\frac{1}{2} \frac{d}{d t} J_{N} \leq-\nu \frac{J_{N}^{1+\frac{1}{p}}}{J_{N-p}^{\frac{1}{p}}}+C_{N, \alpha}\|\nabla \overline{\boldsymbol{u}}\|_{\infty} J_{N}+C \nu \ell^{-2} \operatorname{Re}(\ln R e) J_{N}
$$

and, for $N=0$,

$$
\frac{1}{2} \frac{d}{d t} J_{0} \leq-\nu J_{1}+C \nu \ell^{-2} R e(\ln R e) J_{0} .
$$

When $\alpha \rightarrow 0, J_{N}$ tends to $F_{N}=H_{N}+\tau^{2} \Phi_{N}$, and the result of Theorem 5.1 is consistent with the result achieved for the Navier-Stokes equations in [3].

To obtain length scales estimates, let us define the quantities

$$
\kappa_{N, r}=\left(\frac{J_{N}}{J_{r}}\right)^{\frac{1}{2(N-r)}} .
$$


In the $\alpha \rightarrow 0$ limit, $\kappa_{N, 0}$ behaves as the $2 N$ th moment of the energy spectrum. The aim of this subsection is to find an estimate for the length scales associated with the higher order moments solution $\kappa_{N, 0}(N \geq 2)$. To this end, we first find upper bounds for $\left\langle\kappa_{N, r}^{2}\right\rangle,\left\langle\kappa_{1,0}^{2}\right\rangle$, and $\left\langle\|\nabla \overline{\boldsymbol{u}}\|_{\infty}\right\rangle$. Then we use the identity

$$
\kappa_{N, 0}^{2}=\kappa_{N, 1}^{2(N-1) / N} \kappa_{1,0}^{2 / N}
$$

in order to deduce the result.

(a) The first two bounds are obtained by dividing by $J_{N}$ in Theorem 5.1 and time averaging to obtain

$$
\left\langle\kappa_{N, r}^{2}\right\rangle \leq C_{N, \alpha} \nu^{-1}\left\langle\|\nabla \overline{\boldsymbol{u}}\|_{\infty}\right\rangle+C \ell^{-2} \operatorname{Re}(\ln R e)
$$

and

$$
\left\langle\kappa_{1,0}^{2}\right\rangle \leq C \ell^{-2} \operatorname{Re}(\ln R e) .
$$

Remark 5.1. Note that the bound on $\left\langle\|\overline{\boldsymbol{u}}\|_{\infty}^{2}\right\rangle$ is found to satisfy (see in [10] for more details)

$$
\left\langle\|\overline{\boldsymbol{u}}\|_{\infty}^{2}\right\rangle \leq C \ell^{-2} \nu^{2} V_{\alpha} R e^{11 / 4}
$$

where

$$
V_{\alpha}:=\left({\frac{L}{(\ell \alpha)^{1 / 2}}}^{3}\right)
$$

(b) It is also possible to estimate $\left\langle\|\nabla \overline{\boldsymbol{u}}\|_{\infty}\right\rangle$ by returning to the Equation (1.1) and taking a different way. We take $\boldsymbol{u}=-\alpha^{2} \Delta \overline{\boldsymbol{u}}+\overline{\boldsymbol{u}}$ as test function, then integrating by parts (see Lemma 3.1), using (3.10) and time averaging, to obtain

$$
\begin{aligned}
\nu\left\langle\overline{H_{1}}+2 \alpha^{2} \overline{H_{2}}+\alpha^{4} \overline{H_{3}}\right\rangle & \leq C\left\langle\|\nabla \overline{\boldsymbol{u}}\|_{L^{2}}\|\Delta \overline{\boldsymbol{u}}\|_{L^{2}}^{2}\right\rangle+\left(1+\alpha^{2} \ell^{-2}\right)\left\langle{\overline{H_{0}}}^{1 / 2} \Phi_{0}{ }^{1 / 2}\right\rangle \\
& \leq C\left\langle\|\Delta \overline{\boldsymbol{u}}\|_{L^{2}}^{2}\right\rangle\|\overline{\boldsymbol{u}}\|_{L^{\infty}\left([0, T], H^{1}\right)}+\left(1+\alpha^{2} \ell^{-2}\right)\left\langle{\overline{H_{0}}}^{1 / 2} \Phi_{0}{ }^{1 / 2}\right\rangle \\
& \leq C \alpha^{-2} \nu^{2} \ell^{-4} L^{3} \operatorname{Re}^{3} G r^{2}+C\left(1+\alpha^{2} \ell^{-2}\right) \nu^{3} \ell^{-4} L^{3} R e^{3} .
\end{aligned}
$$

Thus we can write

$$
\left\langle\overline{H_{3}}\right\rangle \leq C(\alpha, \nu, \ell, L) R e^{7} .
$$

This can be used to find the estimate for $\left\langle\|\nabla \overline{\boldsymbol{u}}\|_{\infty}\right\rangle$. In fact, Agmon's inequality [6]

$$
\|\boldsymbol{u}\|_{\infty} \leq\|\boldsymbol{u}\|_{H^{1}}^{1 / 2}\|\boldsymbol{u}\|_{H^{2}}^{1 / 2}
$$

says that

$$
\begin{aligned}
\left\langle\|\nabla \overline{\boldsymbol{u}}\|_{\infty}\right\rangle & \leq\left\langle\overline{H_{3}}\right\rangle^{1 / 4}\left\langle\overline{H_{2}}\right\rangle^{1 / 4} \\
& \leq C(\alpha, \nu, \ell, L) R e^{5 / 2} .
\end{aligned}
$$

(c) Thus we obtain from (5.4) and (5.9) that

$$
\ell^{2}\left\langle\kappa_{N, r}^{2}\right\rangle \leq C(\alpha, \nu, \ell, L) R e^{5 / 2}+C R e(\ln R e) .
$$


In particular, for $r=0$,

$$
\ell^{2}\left\langle\kappa_{N, 0}^{2}\right\rangle \leq C(\alpha, \nu, \ell, L) R e^{5 / 2}+C R e(\ln R e) .
$$

By choosing $r=1$ we can then get an improvement for $\left\langle\kappa_{N, 0}^{2}\right\rangle$ by writing

$$
\begin{aligned}
\left\langle\kappa_{N, 0}^{2}\right\rangle & =\left\langle\kappa_{N, 1}^{2(N-1) / N} \kappa_{1,0}^{2 / N}\right\rangle \\
& \leq\left\langle\kappa_{N, 1}^{2}\right\rangle^{(N-1) / N}\left\langle\kappa_{1,0}^{2}\right\rangle^{1 / N},
\end{aligned}
$$

and then using the above estimates for $\left\langle\kappa_{N, 1}^{2}\right\rangle$ and $\left\langle\kappa_{1,0}^{2}\right\rangle$ which give, for $N \geq 2$,

$$
\ell^{2}\left\langle\kappa_{N, 0}^{2}\right\rangle \leq C(\alpha, \nu, \ell, L)^{(N-1) / N} R e^{5 / 2-3 / 2 N}(\ln R e)^{1 / N}+C R e \ln R e .
$$

Note that when $N=1$ we return to $\ell^{2}\left\langle\kappa_{1,0}^{2}\right\rangle \leq C R e \ln R e$.

5.2. Proof of inequality (1.11). Let us come back to relation (1.3); when $N=0$, we get the energy inequality (3.11):

$$
\frac{d}{d t}\left(\overline{H_{0}}+\alpha^{2} \overline{H_{1}}\right) \leq-\nu\left(\overline{H_{1}}+\alpha^{2} \overline{H_{2}}\right)+{\overline{H_{0}}}^{1 / 2} \Phi_{0}{ }^{1 / 2} .
$$

The Poincaré inequality together with the Cauchy-Schwarz, Young, and Grönwall inequalities in (5.14) imply that $\overline{H_{0}}+\alpha^{2} \overline{H_{1}}$ is uniformly bounded in time according to (3.7). Time averaging, using the fact that the time average of the time derivative in (5.14) vanishes, we obtain

$$
\begin{aligned}
\left\langle\overline{H_{1}}+\alpha^{2} \overline{H_{2}}\right\rangle & \leq\left\langle{\overline{H_{0}}}^{1 / 2} \Phi_{0}{ }^{1 / 2}\right\rangle \\
& \leq c \nu^{2} \ell^{-4} L^{3} R e^{3} .
\end{aligned}
$$

Thus

$$
\left\langle\overline{H_{1}}\right\rangle \leq c \nu^{2} \ell^{-4} L^{3} R e^{3}
$$

and

$$
\left\langle\overline{H_{2}}\right\rangle \leq c \alpha^{-2} \nu^{2} \ell^{-4} L^{3} R e^{3} .
$$

We deduce from (5.16) that the energy dissipation rate $\epsilon=\nu\left\langle\|\nabla \overline{\boldsymbol{u}}\|_{L^{2}}^{2}\right\rangle L^{-3}$ is bounded above by

$$
\epsilon \leq c \nu^{3} \ell^{-4} R e^{3},
$$

and the inverse Kolmogorov length $\lambda_{k}^{-1}=\left(\epsilon / \nu^{3}\right)^{1 / 4}$ is bounded above by

$$
\ell \lambda_{k}^{-1} \leq c R e^{3 / 4}
$$

From (5.17) and by using the interpolation inequality

$$
\overline{H_{N}} \leq{\overline{H_{N-s}}}^{\frac{r}{r+s}}{\overline{H_{N+r}}}^{\frac{s}{r+s}},
$$

that is

$$
\overline{H_{1}} \leq{\overline{H_{0}}}^{\frac{1}{2}}{\overline{H_{2}}}^{\frac{1}{2}}
$$


and we can improve (5.16) in order to obtain

$$
\left\langle\overline{H_{1}}\right\rangle \leq\left\langle\overline{H_{0}}\right\rangle^{\frac{1}{2}}\left\langle\overline{H_{2}}\right\rangle^{\frac{1}{2}} \leq c \alpha^{-1} \nu^{2} \ell^{-3} L^{3} R e^{5 / 2} .
$$

This improves the above result (5.19) for the inverse Kolmogorov length to

$$
\ell \lambda_{k}^{-1} \leq c\left(\frac{\ell}{\alpha}\right)^{1 / 4} R e^{5 / 8}
$$

We also deduce that the energy dissipation rate $\epsilon=\nu\left\langle\|\nabla \overline{\boldsymbol{u}}\|_{L^{2}}^{2}\right\rangle L^{-3}$ is also bounded by $R e^{5 / 2}$, but all of the improved estimates blow up when $\alpha$ tends to zero.

5.3. Proof of inequality (1.12). The authors in [8] showed the existence of a global attractor $\mathcal{A}$ to this model and they made estimates of the fractal dimension of this attractor. The sharp estimate found in [8] for the fractal dimension of $\mathcal{A}$ expressed in terms of Grashof number $G r$ is

$$
d_{F, M L-\alpha}(\mathcal{A}) \leq c\left(\frac{2 \pi}{L \alpha^{2} \gamma}\right)^{3 / 4} G r^{3 / 2}
$$

where

$$
\frac{1}{\gamma}=\min \left(1, \frac{2 \pi}{\alpha^{2} L}\right)
$$

However, the estimate (5.24) can be reexpressed in terms of Re (compare to [10, Section 3]). We observe that their bound for the attractor dimension in [8] depends upon $\left\langle\overline{H_{1}}+\alpha^{2} \overline{H_{2}}\right\rangle$, for which the upper bound is $R e^{3}$. With this observation used in [10], it is found that the estimate of $d_{F, M L-\alpha}(\mathcal{A})$ in [8] converts to

$$
d_{F, M L-\alpha}(\mathcal{A}) \leq c\left(\frac{L^{3 / 2}(2 \pi)^{3 / 2} \ell^{-4}}{\alpha^{2}}\right)^{3 / 4} R e^{9 / 4}
$$

In term of degrees of freedom, the result (5.25) says that $R e^{3 / 4} \times R e^{3 / 4} \times R e^{3 / 4}$ resolution grid points are needed.

Acknowledgment. I would like to thank Professor R. Lewandowski and Professor J.D. Gibbon for helpful remarks and suggestions.

\section{REFERENCES}

[1] A. Cheskidov, D.D. Holm, E. Olson, and E.S. Titi, On a Leray- $\alpha$ model of turbulence, Royal Society London, Proceedings, Series A, Mathematical, Physical and Engineering Sciences, 461, 629-649, 2005.

[2] C. Doering and C. Foias, Energy dissipation in body-forced turbulence, J. Fluid Mech., 467, 289-306, 2002.

[3] C. Doering and J. Gibbon, Applied Analysis of the Navier-Stokes Equations, Cambridge University Press, 1995.

[4] C.R. Doering and E.S. Titi, Exponential decay rate of the power spectrum for solutions of the Navier-Stokes equations, Physical Fluids, 7, 1384-1390, 1995.

[5] C. Foias, D. Holm, and E.S. Titi, The three dimensional viscous Camassa-Holm equations and their relation to the Navier-Stokes equations and turbulence theory, J. Dyn. Diff. Equ., 14, $1-35,2002$.

[6] C. Foias, O. Manley, R. Rosa, and R. Temam, Navier-Stokes Equations and Turbulence, Cambridge University Press, 2001. 
[7] U. Frisch, Turbulence: The Legacy of A.N. Kolomogrov, Cambridge University Press, 1995.

[8] A.A. Ilyin, E.M. Lunasin, and E.S. Titi, A modified Leray-alpha subgrid-scale model of turbulence, Nonlin., 19, 879-897, 2006.

[9] J.D. Gibbon and C.R. Doering, Intermittency and regularity issues in 3d Navier-Stokes turbulence, Arch. Rat. Mech. Anal., 177, 115-150, 2006.

[10] J.D. Gibbon and D.D. Holm, Length-scale estimates for the LANS- $\alpha$ equations in terms of the Reynolds number, Phys. D, 220, 69-78, 2006.

[11] J.D. Gibbon and D.D. Holm, Estimates for the LANS- $\alpha$, Leray- $\alpha$ and Bardina models in terms of a Navier-Stokes Reynolds number, Indiana U. Math. J., 57, 2761-2773, 2008.

[12] D.A. Jones and E.S. Titi, Upper bounds for the number of determining modes, nodes and volumes elements for the Navier-Stokes equations, Indiana U. Math. J., 42, 875-887, 1993.

[13] W. Layton and R. Lewandowski, On a well posed turbulence model, Cont. Dyn. Sys. Series B, 6, 111-128, 2006.

[14] R. Lewandowski, On a continuous deconvolution equation for turbulence models, Lecture Notes of Necas Center for Mathematical Modeling, 5, 62-102, 2009.

[15] R. Temam, Infinite-Dimensional Dynamical Systems in Mechanics and Physics, SpringerVerlag, New York, 68, 1988. 